\title{
Bronchoalveolar lavage in suspected COVID-19 cases with a negative nasopharyngeal swab: a retrospective cross-sectional study in a high-impact Northern Italy area
}

\author{
Caterina Barberi ${ }^{1,2}$. Elena Castelnuovo ${ }^{1,2} \cdot$ Andrea Dipasquale $^{1,3} \cdot$ Federica Mrakic Sposta $^{4} \cdot$ Giulia Vatteroni $^{1,4}$. \\ Lorenzo Maria Canziani ${ }^{1,3}$. Marco Alloisio ${ }^{1,5}$. Michele Ciccarelli ${ }^{1,6}$. Carlo Selmi ${ }^{1,7}$. Giorgio Maria Ferraroli ${ }^{1,5}$
}

Received: 6 December 2020 / Accepted: 14 March 2021 / Published online: 26 March 2021

(c) Società Italiana di Medicina Interna (SIMI) 2021

\begin{abstract}
COVID-19 diagnosis relies on molecular testing for SARS-CoV-2 via nasopharyngeal swab in the presence of suggestive clinical, radiological and laboratory findings. Since bronchoalveolar lavage liquid (BAL) collected during fibrobronchoscopy may increase test sensitivity compared to nasopharyngeal swabs, it was performed during the 2020 pandemic in clinically or radiologically suspected cases. Our aim was to determine whether clinical features, chest computed tomography (CT) findings or laboratory tests may predict patients testing positive for SARS-CoV-2 at BAL after a negative nasopharyngeal swab. We performed a retrospective cross-sectional study with multivariable analysis of suspected patients who were tested for SARS-CoV-2 at BAL after at least one negative nasopharyngeal swab. Univariable logistic regression for odds ratio and multivariate models was calculated to determine clinical, radiological and laboratory predictors. 32/198 (16\%) patients had BAL positive for SARS-CoV-2, while 65/198 tested positive for other pathogens at BAL. Of the 32 patients positive for COVID, 4 had a coinfection at BAL, being thus positive both for COVID as well as for another pathogen while the remaining 105 patients were negative for COVID and other pathogens at BAL. COVID-19 patients had more often highly suggestive CT findings, higher number of involved lobes, more often ground glass opacity of more than $50 \%$ of lung parenchyma, and less frequently other radiologically suspected infections. At multivariate model, temperature also predicted BAL positivity. The procedure was well tolerated - with only one desaturation episode-while no healthcare worker was infected. In conclusion, when nasopharyngeal swabs are negative but there is clinical or imaging suspicion of COVID-19, BAL represents a complementary diagnostic tool, particularly in conjunction with suggestive/more extensive lung involvement at CT scan. The procedure did not carry increased risks for patients nor for operators, while allowing to free hospital resources, avoiding unnecessary isolations.
\end{abstract}

Keywords Bronchoscopy $\cdot$ Bronchoalveolar lavage $\cdot$ Coronavirus infections $\cdot$ Pandemics $\cdot$ Interstitial pneumonia $\cdot$ Chest computed tomography

\section{Introduction}

During the COVID-19 pandemic, several studies have been conducted to identify the best methods for the detection of SARS-CoV-2, the pathogen responsible for the disease. Considering its fast spreading, also related to the presence

Caterina Barberi and Elena Castelnuovo contributed equally to this work.

Carlo Selmi

carlo.selmi@hunimed.eu

Extended author information available on the last page of the article of asymptomatic individuals, diagnosis could not rely on clinical picture alone $[1,2]$. In patients manifesting consistent symptoms, the molecular diagnosis of SARS-CoV-2 infection is largely based on nasopharyngeal swabs $[1,3,4]$ with an overall $63 \%$ sensitivity $[5,6]$, which varies over the disease stages. To reduce the rate of false negative results, the need to collect two specimens has been advised pending local feasibility [4]. Additionally, a suggestive pattern at chest computed tomography (CT) manifests a high sensitivity (97\%), but remains poorly specific (25\%) [7]. Consequently, when SARS-CoV-2 was not confirmed by means of nasopharyngeal swabs, the dilemma of whether still treating 
and considering the patients as COVID-19 posed many organizational and therapeutic concerns.

Since SARS-CoV-2 has been isolated from numerous biological fluids [8-10], the analysis of bronchoalveolar lavage (BAL) represents an additional method for the virus detection $[2,11,12]$ with reported molecular sensitivity rates as high as $93 \%$ [13]. Based on these observations, we hypothesize that obtaining BAL could be helpful in patients with a negative nasopharyngeal swab and a suggestive CT pattern and/or subclinical picture. This is in apparent conflict with the current recommendations suggesting that fibrobronchoscopy (FBS) with BAL collection for COVID-19 should be considered in case of emergency and in immunocompromised patients [3] or when a coexisting infectious disease is suspected $[1,13,14]$, particularly due to the possible procedure-related risks for the operator and the patient [15]. On the other hand, considering the critical outbreak settings in our region, a misdiagnosis of a COVID-19 patient may endanger public health and may prevent patients being appropriately treated.

In the present study, we took advantage of the decision our center made to obtain BAL via FBS in all patients presenting with a suspicion of COVID-19 and at least one negative nasopharyngeal swab, for which no absolute contraindications were observed at a multidisciplinary approach. We performed a retrospective analysis of clinical, radiological and laboratory data of these patients to determine the baseline differences between those testing positive and negative at BAL for SARS-CoV-2. We further collected data on the complications observed and on the risks of healthcare workers related to the exposure to airdrops during the procedure.

\section{Materials and methods}

\section{Subjects}

We performed a retrospective cross-sectional study including all patients admitted to Humanitas Research Hospital-Rozzano-from March 1, 2020 until April 30, 2020, who underwent FBS with BAL collection for the research of SARS-CoV-2. This period was selected considering the high incidence of the disease in Italy; from the data of May 4, 2020 [16], the number of cases was 210.717, the estimated cumulative incidence per 100.000 inhabitants 348.5 [17].

All patients tested negative at one or more nasopharyngeal swabs (SARS-CoV-2 Assay Allplex ${ }^{\mathrm{TM}}$ ). The choice to perform FBS was made following a multidisciplinary approach, which included infectious diseases specialists, pulmonologists, and thoracic surgeons in all cases. For all patients, we collected baseline characteristics, based on the major features reported in the literature $[1,11]$, including age and sex, symptoms (dyspnea, cough, digestive, dysgeusia, and anosmia), signs (fever, $\mathrm{SpO}_{2}<92 \%, \mathrm{PaO}_{2}: \mathrm{FiO}_{2}$ ), chest $\mathrm{CT}$ scan findings (number of involved lobes, ground glass opacity-GGO- $>50 \%$, consolidation $>50 \%$, radiological report suggestive for a non-COVID infection, radiological grading as explained below), laboratory tests (absolute white blood cells, lymphocytes, eosinophils, serum levels of lactate dehydrogenase, C-reactive protein, procalcitonin, D-dimer, fibrinogen, ferritin, and pneumococcal urinary antigen) and department in which the patient underwent FBS (internal medicine, intensive care unit-ICU-, surgery, onco-hematology, neurology). All clinical, imaging, and laboratory variables were registered and measured either in the emergency department or when a high clinical suspicion arose during hospitalization. In case of surgical patients, BAL execution was highly recommended in the presence of any clinical or radiological suspicions, for the adequate management of the patient, including the use of dedicated operatory theater and ward as well as dedicated healthcare personnel. In cases of elective surgery, therefore, we considered the pre-operative laboratory tests and radiological examinations.

\section{Imaging}

Based on COVID-19 Reporting and Data System (CORADS) classification [18], we adapted the grading of chest CT scan to simplify the report and to facilitate the communication with treating physicians, encompassing Grade 0 (negative for COVID - corresponding to CO-RADS 1-2), Grade 1 (doubtful for COVID-corresponding to CO-RADS 2-3), Grade 2 (suggestive for COVID-corresponding to CO-RADS 4-5). For the purpose of this study, all CT scans were reviewed by two independent radiologists (at least one expert radiologist, $>15$ years of experience), who were blind to the result of the BAL tests.

\section{FBS and BAL collection}

After the informed consent was signed by each patient, FBS was performed with a single use flexible bronchoscope $\left(\mathrm{Ambu}^{\circledR}{ }^{\circledR} \mathrm{aScope}^{\mathrm{TM}} 4\right.$ Broncho Regular 5.0/2.2) generally via a transnasal route. When driven by imaging, BAL fluid was collected in the site most suggestive for inflammation/infection at CT scan; when CT scan was negative, but symptoms were suggestive, samples were taken arbitrarily. In all cases, specimens were collected in disposable sterile containers (Argyle ${ }^{\mathrm{TM}}$ Specimen Trap-40 ml) and sent to the laboratory for immediate search of SARS-CoV-2 (SARS-CoV-2 Assay Allplex ${ }^{\mathrm{TM}}$ ) and other pathogens, when appropriate. $20 \mathrm{cc}$ of sterile saline $(0.9 \% \mathrm{NaCl}$ without additives) were used as instilling fluid, while 5-7 cc were aspirated.

When a non-COVID-19 infection was suspected, a wider panel of microbiological tests included PCR tests for 
influenza $\mathrm{A}$, influenza $\mathrm{B}$, respiratory syncytial virus, coronavirus (not COVID-19), parainfluenza virus, Metapneumovirus, bocavirus, Enterovirus, rhinovirus, adenovirus and $M$. tuberculosis; cultural research of bacteria and fungi. In case of immunocompromised patients, the research for opportunistic pathogens was also performed.

Mild sedation with IV midazolam and local anesthesia with lidocaine were administered. $\mathrm{SpO}_{2}$ was monitored using a peripheral pulse oximeter throughout the procedure and a thorough cleaning of surfaces was performed after every procedure. All procedures were performed by a thoracic surgeon supported by a nurse of the ward where the patient was located; both were wearing disposable personal protective equipment (PPE), following the standard donning and doffing protocol [1]: face shield, eye-shields, FFP3 mask, appropriate gown, gloves and overshoes. In all cases, FBS were performed in a negative pressure room.

\section{Statistical analysis}

Categorical variables were reported as number (percentage) and continuous variables as median (interquartile rangeIQR). Listwise deletion was used for dealing with missing data. Chi-square test and Mann-Whitney test were used accordingly to the type of variable. Odds ratio (OR) was calculated by means of univariable logistic regression, using BAL positivity as outcome. All tests were leveled for significance at $p$ value $<0.05$. Multivariate models to estimate OR used BAL positivity for SARS-CoV-2 as outcome, and variables were selected if the $p$ value was $<0.1$ at univariable logistic regression and with less than $30 \%$ of missing data. The logistic regression was performed with a stepwise approach. Data were analyzed with STATA version 16.0 (StataCorp LLC, College Station, TX).

\section{Results}

We included 198 patients who underwent BAL for the research of SARS-CoV-2 after at least one negative nasopharyngeal swab and a suggestive clinical or radiological picture. 12/198 patients underwent two nasopharyngeal swabs, 1/198 underwent three nasopharyngeal swabs, while the majority of our patients (185/198) underwent one nasopharyngeal swab before performing BAL collection, largely based on internal recommendations and operating strategies. The baseline characteristics of the study cohort are reported in Table 1. The overall rate of detection of SARS-CoV-2 at BAL was $16 \%$. The $32(16 \%)$ patients positive for SARS$\mathrm{CoV}-2$ at BAL were more often men (72 vs. $51 \%, p=0.027$ ) and younger [63 (IQR 46-74.5) vs. 71 (IQR 60-79) years, $p=0.014]$, had more often dysgeusia and anosmia (15 vs.
$3 \%, p=0.003)$ and higher fever $\left[37.7{ }^{\circ} \mathrm{C}\right.$ (IQR 37.2-38.3) vs. $37.0{ }^{\circ} \mathrm{C}$ (IQR $\left.\left.36.5-37.8\right), p=0.002\right]$.

The radiological findings were markedly different between patients testing SARS-CoV-2 positive or negative at BAL, with the former having a higher number of involved lobes [5 (IQR 1-5) vs. 2 (IQR 1-4), $p=0.007$ ], more often a ground glass opacity involving over $50 \%$ of the lung parenchyma (64 vs. $42 \%, p=0.024$ ), less frequently signs of nonCOVID-19 infections as defined by the radiologist (19 vs. $62 \%, p<0.001$ ), and a more frequent highly suggestive radiological grading (65 vs. $23 \%$. $p<0.001)$. Nonetheless, $5 / 54$ (9\%) patients with CT scan with grade 0 were positive for SARS-CoV-2 at BAL. Among laboratory tests, patients testing SARS-CoV-2 positive at BAL had higher serum LDH [318 (IQR 262-372) vs. 243 (IQR 185-329) IU/l, $p=0.002$ ].

Table 2 illustrates the univariable and multivariable logistic regression analyses to predict SARS-CoV-2 positivity at BAL. In univariable models, male sex (OR 2.49, 95\% CI 1.09-5.71, $p=0.031$ ), ear-nose-throat (ENT) symptoms (OR $6.57,95 \%$ CI 1.66-26.07, $p=0.007$ ), CT features including the number of involved lobes at CT (OR 1.35, 95\% CI $1.09-1.66, p=0.006$ ), ground grass opacity over $50 \%$ (OR $2.46,95 \%$ CI $1.11-5.48, p=0.027)$ and radiological grade 2 (OR 5.16, 95\% CI 1.77-15.00, $p=0.003$ ) predicted SARSCoV-2 positivity at BAL, while the opposite was observed for older age (OR 0.97, 95\% CI 0.95-0.99, $p=0.014$ ) and radiological evidence of a non-COVID infection (OR 0.14, 95\% CI 0.06-0.38, $p<0.001)$. With multivariable model, a total of 173 observations were used (87\%) due to listwise deletion. Fever (OR 1.94 per additional ${ }^{\circ} \mathrm{C}$, 95\% CI $1.13-3.33, p=0.016$ ) and $\mathrm{CT}$ radiological grade 2 (OR 7.36, 95\% CI 2.10-25.77, $p=0.002$ ) predicted positivity at BAL, while radiological evidence of a non-COVID infection (OR $0.12,95 \%$ CI $0.04-0.41, p=0.001)$ predicted SARS-CoV-2 negativity at BAL. In our study population, 65/198 BAL turned positive for the research of other pathogens and the isolated pathogens are listed in Supplementary Table 1. Considering the four patients who were coinfected (meaning that they were positive both for COVID as well as for another pathogen-bacterial, viral or fungal), BAL allowed to identify the causative agent in $93 / 198$ of cases. The remaining $105 / 198$ patients were negative for the search of any tested pathogen at BAL.

\section{Discussion}

Our retrospective cross-sectional study included all patients admitted to Humanitas Research Hospital-Rozzano-from March 1, 2020 until April 30, 2020, a timeframe selected considering the high incidence of the disease in Italy [16, 17]. According to the gathered displayed data with the chosen statistical analysis, we believe that our study findings 
Table 1 Demographic and clinical characteristics of patients included in the study

\begin{tabular}{|c|c|c|c|c|c|}
\hline & $\begin{array}{l}\text { Number of } \\
\text { observations }\end{array}$ & Total $(n=198)$ & Positive BAL $(n=32)$ & Negative BAL $(n=166)$ & $p$ value \\
\hline Male sex $(n)$ & $198(100 \%)$ & $107(54 \%)$ & $23(72 \%)$ & $84(51 \%)$ & 0.027 \\
\hline Age (years) & $198(100 \%)$ & $70(58-78)$ & $63(46-74.5)$ & $71(60-79)$ & 0.014 \\
\hline Unit & $198(100 \%)$ & & & & \\
\hline Internal medicine & & $146(74 \%)$ & $27(84 \%)$ & $119(72 \%)$ & 0.078 \\
\hline ICU & & $3(1 \%)$ & $0(0 \%)$ & $3(2 \%)$ & \\
\hline Surgery & & $19(10 \%)$ & $5(16 \%)$ & $14(8 \%)$ & \\
\hline Onco-hematology & & $12(6 \%)$ & $0(0 \%)$ & $12(7 \%)$ & \\
\hline Neurology & & $18(9 \%)$ & $0(0 \%)$ & $18(11 \%)$ & \\
\hline \multicolumn{6}{|l|}{ Symptoms and respiratory function } \\
\hline Dyspnea & $177(89 \%)$ & $72(41 \%)$ & $17(55 \%)$ & $55(38 \%)$ & 0.077 \\
\hline Cough & $178(90 \%)$ & $58(33 \%)$ & $12(38 \%)$ & $46(32 \%)$ & 0.512 \\
\hline Gastrointestinal & $178(90 \%)$ & $18(10 \%)$ & $5(16 \%)$ & $13(9 \%)$ & 0.253 \\
\hline Dysgeusia, anosmia & $178(90 \%)$ & $9(5 \%)$ & $5(15 \%)$ & $4(3 \%)$ & 0.003 \\
\hline Temperature & $179(90 \%)$ & $37.2(36.5-38)$ & $37.7(37.2-38.3)$ & $37(36.5-37.8)$ & 0.002 \\
\hline $\mathrm{SpO}_{2}<92 \%$ & $176(89 \%)$ & $56(32 \%)$ & $11(35 \%)$ & $45(31 \%)$ & 0.629 \\
\hline $\mathrm{PaO}_{2}: \mathrm{FiO}_{2}$ & $122(61 \%)$ & $307(254-362)$ & $312(255-347)$ & $302(248-376)$ & 0.510 \\
\hline \multicolumn{6}{|l|}{ CT findings } \\
\hline Number of involved lobes & $196(99 \%)$ & $2(1-5)$ & $5(1-5)$ & $2(1-4)$ & 0.007 \\
\hline Ground glass opacity $>50 \%$ & $196(99 \%)$ & $90(46 \%)$ & $20(64 \%)$ & $70(42 \%)$ & 0.024 \\
\hline Consolidation & $196(99 \%)$ & $64(33 \%)$ & $8(26 \%)$ & $56(34 \%)$ & 0.395 \\
\hline Non-COVID infection* & $196(99 \%)$ & $108(55 \%)$ & $6(19 \%)$ & $102(62 \%)$ & $<0.001$ \\
\hline \multicolumn{6}{|l|}{ Conclusion } \\
\hline Negative & $196(99 \%)$ & $54(27 \%)$ & $5(16 \%)$ & $49(30 \%)$ & $<0.001$ \\
\hline Borderline & & $84(43 \%)$ & $6(19 \%)$ & $78(47 \%)$ & \\
\hline Suggestive & & $58(30 \%)$ & $20(65 \%)$ & $38(23 \%)$ & \\
\hline \multicolumn{6}{|l|}{ Laboratory tests } \\
\hline White blood cells $\left(10^{3} / \mu \mathrm{l}\right)$ & $198(100 \%)$ & $9.0(6.2-12.7)$ & $7.5(5.4-12.9)$ & $9.0(6.3-12.5)$ & 0.488 \\
\hline Lymphocytes $\left(10^{3} / \mu \mathrm{l}\right)$ & $198(100 \%)$ & $1.0(0.6-1.7)$ & $1.0(0.7-1.3)$ & $1.1(0.6-1.7)$ & 0.400 \\
\hline Eosinophils $\left(10^{3} / \mu \mathrm{l}\right)$ & $198(100 \%)$ & $0(0-0.1)$ & $0(0-0.1)$ & $0(0-0.1)$ & 0.003 \\
\hline Lactate dehydrogenase (IU/l) (ULN 248) & $177(89 \%)$ & $254(204-347)$ & $318(262-372)$ & $243(185-329)$ & 0.002 \\
\hline C-reactive protein (mg/dl) (ULN 0.5) & $197(99 \%)$ & $5.7(1.6-12.6)$ & $8.15(4.8-12.25)$ & $4.87(1.3-13.4)$ & 0.068 \\
\hline Procalcitonin (ng/ml) (ULN 0.5) & $165(83 \%)$ & $0.17(0.07-0.68)$ & $0.17(0.10-0.34)$ & $0.16(0.07-0.72)$ & 0.960 \\
\hline D-Dimer (ng/ml) (ULN 500) & $143(72 \%)$ & $571(323-1092)$ & $522(278-1005)$ & $620(330-1105)$ & 0.311 \\
\hline Fibrinogen (mg/dl) (ULN 400) & $143(72 \%)$ & $524(380-665)$ & $583(445-676)$ & $520(363-663)$ & 0.269 \\
\hline Ferritin (ng/ml) (ULN 336) & $91(46 \%)$ & $370(105-870)$ & $485(180-990)$ & $353(93-691)$ & 0.054 \\
\hline Pneumococcal urinary antigen & $171(86 \%)$ & $35(20 \%)$ & $9(30 \%)$ & $26(18 \%)$ & 0.154 \\
\hline
\end{tabular}

Continuous variables are expressed as median \pm interquartile range (IQR)

$U L N$ upper limit of normal

*At CT scan

have several important implications for the management of COVID-19.

First, the most relevant observation from our study was the agreement between the radiological pattern and the BAL positivity for COVID-19. Indeed, when CT scan presented with evidence of ground glass opacity $>50 \%$ (64 vs. $42 \%$ ) and higher number of lobes involved (5 vs. 2 ), the research for COVID-19 in BAL was likely to be positive.
This observation could be explained by the fact that FBS and specimen collection normally take into account the localization of ground glass opacity and/or areas around the bronchial tree where major inflammation is visualized. By doing so, the likelihood of isolating the pathogen in BAL obviously increases. The positive agreement between CT scan and BAL results observed in a large number of patients is in contrast with the findings from 28 patients [13], that is that 
Table 2 Univariable and multivariable logistic regression analysis for BAL SARS-CoV-2 positivity

\begin{tabular}{|c|c|c|c|c|}
\hline & $\begin{array}{l}\text { Univariable } \\
\text { OR }(95 \% \mathrm{CI})\end{array}$ & $p$ value & $\begin{array}{l}\text { Multivariable } \\
\text { OR }(95 \% \text { CI })\end{array}$ & $p$ value \\
\hline Male sex (vs. female) & $2.49(1.09-5.71)$ & $\mathbf{0 . 0 3 1}$ & $1.52(0.52-4.41)$ & 0.440 \\
\hline Age (per year) & $0.97(0.95-0.99)$ & 0.014 & $0.98(0.95-1.01)$ & 0.193 \\
\hline \multicolumn{5}{|l|}{ Symptoms and respiratory function } \\
\hline Dyspnea & $2.01(0.92-4.39)$ & 0.081 & & \\
\hline Cough & $1.30(0.59-2.89)$ & 0.513 & & \\
\hline Gastrointestinal & $1.89(0.62-5.76)$ & 0.260 & & \\
\hline ENT (dysgeusia, anosmia) & $6.57(1.66-26.07)$ & 0.007 & $4.51(0.81-25.08)$ & 0.085 \\
\hline Temperature (per $1^{\circ} \mathrm{C}$ ) & $1.99(1.29-3.09)$ & 0.002 & $1.94(1.13-3.33)$ & 0.016 \\
\hline Desaturation $\left(\mathrm{SpO}_{2}<92 \%\right)$ & $1.22(0.54-2.76)$ & 0.630 & & \\
\hline $\mathrm{PaO}_{2}: \mathrm{FiO}_{2}($ per 25$)$ & $0.97(0.86-1.08)$ & 0.536 & & \\
\hline \multicolumn{5}{|l|}{ CT findings } \\
\hline Number of involved lobes & $1.35(1.09-1.66)$ & 0.006 & & \\
\hline Ground glass opacity $>50 \%$ & $2.46(1.11-5.48)$ & 0.027 & & \\
\hline Consolidation & $0.68(0.28-1.61)$ & 0.378 & & \\
\hline Non-COVID infection* & $0.14(0.06-0.38)$ & $<0.001$ & $0.12(0.04-0.41)$ & 0.001 \\
\hline \multicolumn{5}{|l|}{ Conclusion (vs. negative) } \\
\hline Borderline & $0.75(0.22-2.60)$ & 0.655 & $2.26(0.48-10.61)$ & 0.300 \\
\hline Suggestive & $5.16(1.77-15.00)$ & 0.003 & $7.36(2.10-25.77)$ & 0.002 \\
\hline \multicolumn{5}{|l|}{ Laboratory tests } \\
\hline White blood cells (per $\left.10^{3} / \mu \mathrm{l}\right)$ & $0.98(0.90-1.05)$ & 0.566 & & \\
\hline Lymphocytes (per $\left.10^{3} / \mu \mathrm{l}\right)$ & $0.73(0.43-1.22)$ & 0.230 & & \\
\hline Eosinophils (per $10^{3} / \mu \mathrm{l}$ ) & $0.08(0.00-3.12)$ & 0.179 & & \\
\hline Lactate dehydrogenase (per 100 IU/l) & $1.23(0.96-1.59)$ & 0.104 & & \\
\hline C-reactive protein (per mg/dl) & $1.00(0.96-1.03)$ & 0.984 & & \\
\hline Procalcitonin (ng/ml) & $0.82(0.58-1.15)$ & 0.248 & & \\
\hline D-Dimer (per mg/ml) & $0.93(0.73-1.18)$ & 0.543 & & \\
\hline Fibrinogen (per 100 mg/dl) & $1.06(0.87-1.30)$ & 0.542 & & \\
\hline Ferritin (per mg/ml) & $1.29(0.86-1.92)$ & 0.211 & & \\
\hline Pneumococcal urinary antigen & $1.89(0.78-4.61)$ & 0.159 & & \\
\hline
\end{tabular}

Bold values indicate statistically significant

Odds ratios (OR) are reported with $95 \%$ confidence intervals (95\% CI); in the case of continuous variables, absolute increments corresponding to the OR are specified. For the multivariate analysis, a total of 173 observations were used $(87 \%)$ due to listwise deletion

*At CT scan
SARS-CoV-2 infection can be safely ruled out regardless of a suggestive CT scan. On the other hand, the authors of a recent multicentric study on 131 patients [4] reported that patients suspected for COVID-19 presented with a higher number of radiological alterations.

Second, 5/54 patients who had a grade 0 at CT scan demonstrated BAL positivity for COVID-19. This finding highlights the fact that a negative CT scan cannot rule out with certainty a SARS-CoV-2 infection, especially in the hypothesis that the pathogen has already moved to the lower respiratory tract at the time of FBS, without causing the overt radiological pattern. This may also be related to the fact that some patients presented with non-respiratory symptoms at presentation, thus the disease was active, still in the absence of a pulmonary involvement. In a similar recent multicentric study [2], when CT scan was considered as normal (10 patients), also the BAL for SARS-CoV-2 turned negative, confirming our overall trend. However, when possible, BAL should still be performed to decrease the rate of false negative results and to de-isolate these patients with more safety, even if they represent a restricted group of cases (in our dataset $9 \%$ ).

Third, when a non-COVID-19 pneumonia was suspected at CT scan, BAL was more frequently negative for COVID19: $2 / 108$ patients were $\mathrm{BAL}^{+}$only for COVID-19; 4/108 patients were positive both for COVID-19 as well as for another infection. 65/198 (33\%) turned BAL positive for the research of other pathogens, thus allowing to begin a specific 
therapy when indicated (in case of a low number of copies and/or possible contaminants, no therapy was initiated). This number is slightly lower compared to another similar research, which, however, only included 28 patients [13]. As a fact, BAL allowed to treat patients-especially immunocompromised ones-with a targeted antibiotic therapy, after the pathogen was isolated [3]. Differently, in the multicentric study of Geri et al. [2], no concomitant pulmonary infections were detected, probably because an empirical antibiotic therapy was started a few days in advance before the performance of BAL.

Fourth, BAL-positive patients were younger compared to the negative ones. This may reflect a bias by which older patients with a severe compromised clinical picture and concomitant comorbidities who would have probably turned positive for COVID-19 at BAL did not undergo FBS at all as the risks were considered to outweigh the benefits of a molecular diagnosis. Nonetheless, a BAL negative for SARS-CoV-2 allowed to avoid the unnecessary isolation of suspect cases, thus maximizing the number of patients admitted to the COVID-19 wards and minimizing the risk for healthcare operators. The former organizational change was of major importance, if we consider the critical outbreak setting in our region (Milan-Lombardy) during that particular period, where we needed to treat and hospitalize an enormous number of patients over a short amount of time with a limited number of beds and resources, as occurred in other hospitals in nearby regions [4].

Fifth, during the observation period and the 198 FBS procedures, only one was complicated by desaturation after the procedure and required high-flow oxygen therapy. In that case, FBS demonstrated the presence of a bronchial obstruction due to an advanced malignancy that had not been previously diagnosed. Data on the safety of the healthcare personnel are also reassuring as 4/4 thoracic surgeons performing FBS tested negative for SARS-CoV-2 at nasopharyngeal swab during the course of the study. We may thus surmise that adequate PPE and negative pressure environments allow to reduce the risk for healthcare workers performing FBS, a procedure that had been considered as a high-risk procedure for the viral spreading.

Since BAL is not readily available at all Centers, other specimens collected from the lower respiratory tract have been considered for molecular testing, such as induced sputum. However, this approach has proven to be slightly superior to nasopharyngeal swabs, but less sensitive compared to BAL [6]. The unique advantage of BAL versus other fluids is that it can be performed with a higher precision, possibly in concordance with the radiological findings, consequently increasing diagnostic accuracy. Induced sputum is de facto collected blindly.

We are well aware of the limitations of our study. First and foremost, this is a retrospective and monocentric study, but we acknowledge that the multidisciplinary approach and the rigorous recommendations that were internally followed reduce the risk of bias from the study design.

Second, FBS and BAL were ordered based on different indications and in different settings, not allowing the estimate of a uniform pre-test probability, despite the setting within one of the highest impact COVID-19 geographical regions. As an example, in hematological and oncological patients, BAL was frequently requested to detect nonCOVID-19 pathogens, but during the pandemic the search for SARS-CoV-2 was also included, while the clinical suspicion was minimal in patients for elective surgery. This could in part account for the rate of detection for SARS-CoV-2 in our study being lower than the one reported in a multicenter study [4], in which detection rate was reported to be $32.8 \%$ (43/131 patients). At the same time, our detection rate was in agreement with a previous paper regarding PCR assays identifying viral nucleic acid [19], in which BAL provided a specific diagnosis in $17 \%$ of the cases. Nonetheless, according to our internal recommendations, BAL was performed to rule out with the highest possible degree of certainty the positivity for COVID-19, to reduce the risk of spreading due to asymptomatic carriers and to guarantee a better patient management.

Third, we observed a low number of patients who had at least two negative nasopharyngeal swabs before BAL. The choice of performing one or more nasopharyngeal swabs was ascribed to the Hospital arrangements secondary to multidisciplinary discussion, possibly trying to maximize the chance of COVID-19 identification with a more sensitive method.

Forth, in the statistical model, $13 \%$ of patients were excluded from the multivariable model due to listwise deletion for missing data. In future studies, this point could be improved - for instance-by performing a more standardized set of laboratory tests upon patients' arrival in the hospital.

Ultimately, this study was not intended to determine the sensibility and sensitivity of BAL for the detection of SARS$\mathrm{CoV}-2$ for which dedicated perspective studies are awaited.

\section{Conclusions}

In conclusion, we submit that FBS with BAL should be considered as a complementary diagnostic tool that did not carry an increased risk for the patient or the operator but provided additional information in suspected cases, particularly when associated with fever or a suggestive CT scan.

Supplementary Information The online version contains supplementary material available at https://doi.org/10.1007/s11739-021-02714-y. 
Acknowledgements This work was conducted in the framework of, and made possible by, the collective effort of the Humanitas COVID19 Task Force.

\section{Declarations}

Conflict of interest All authors have completed the uniform disclosure form. The authors have no conflicts of interest to declare. This present research did not receive any specific grant from funding agencies in the public, commercial, or not-for-profit sectors.

Ethics approval This retrospective study involving human participants was in accordance with the ethical standards of the institutional and national research committee and with the 1964 Helsinki Declaration and its later amendments or comparable ethical standards. The Human Investigation Committee (IRB) of the Humanitas Research Hospital approved this study.

Informed consent Informed consent was obtained from all individual participants included in the study.

\section{References}

1. Pritchett MA, Oberg CL, Belanger A, De Cardenas J, Cheng G, Nacheli GC, Franco-Paredes C, Singh J, Toth J, Zgoda M, Folch E (2020) Society for advanced bronchoscopy consensus statement and guidelines for bronchoscopy and airway management amid the COVID-19 pandemic. J Thorac Dis. 12(5):1781-1798. https:// doi.org/10.21037/jtd.2020.04.32

2. Geri P, Salton F, Zuccatosta L, Tamburrini M, Biolo M, Busca A, Santagiuliana M, Zuccon U, Confalonieri P, Ruaro B, D’Agaro P, Gasparini S, Confalonieri M (2020) Limited role for bronchoalveolar lavage to exclude COVID-19 after negative upper respiratory tract swabs: a multicentre study. Eur Respir J. https://doi.org/ 10.1183/13993003.01733-2020

3. Ramos KJ, Kapnadak SG, Collins BF, Pottinger PS, Wall R, Mays JA, Perchetti GA, Jerome KR, Khot S, Limaye AP, Mathias PC, Greninger A (2020) Detection of SARS-CoV-2 by bronchoscopy after negative nasopharyngeal testing: stay vigilant for COVID-19. Respir Med Case Rep. https://doi.org/10.1016/j.rmcr.2020.101120

4. Patrucco F, Albera C, Bellocchia M, Foci V, Gavelli F, Castello LM, Bellan M, Sainaghi PP, Airoldi C, Balbo PE, Solidoro P (2020) SARS-CoV-2 detection on bronchoalveolar lavage: an Italian multicenter experience. Respiration 99(11):970-978. https:// doi.org/10.1159/000511964

5. Wang X, Tan L, Wang X, Liu W, Lu Y, Cheng L, Sun Z (2020) Comparison of nasopharyngeal and oropharyngeal swabs for SARS-CoV-2 detection in 353 patients received tests with both specimens simultaneously. Int J Infect Dis 94:107-109. https:// doi.org/10.1016/j.ijid.2020.04.023

6. Wang W, Xu Y, Gao R, Lu R, Han K, Wu G, Tan W (2020) Detection of SARS-CoV-2 in different types of clinical specimens. JAMA 323(18):1843-1844. https://doi.org/10.1001/jama.2020. 3786

7. Ai T, Yang Z, Hou H, Zhan C, Chen C, Lv W, Tao Q, Sun Z, Xia L (2020) Correlation of chest CT and RT-PCR testing for coronavirus disease 2019 (COVID-19) in China: a report of 1014 cases. Radiology 296(2):E32-E40. https://doi.org/10.1148/radiol.20202 00642
8. Perchetti GA, Nalla AK, Huang ML, Zhu H, Wei Y, Stensland L, Loprieno MA, Jerome KR, Greninger AL (2020) Validation of SARS-CoV-2 detection across multiple specimen types. J Clin Virol 128:104438. https://doi.org/10.1016/j.jcv.2020.104438

9. Sun J, Xiao J, Sun R, Tang X, Liang C, Lin H, Zeng L, Hu J, Yuan R, Zhou P, Peng J, Xiong Q, Cui F, Liu Z, Lu J, Tian J, Ma $\mathrm{W}, \mathrm{Ke} \mathrm{C}$ (2020) Prolonged persistence of SARS-CoV-2 RNA in body fluids. Emerg Infect Dis 26(8):1834-1838. https://doi.org/ 10.3201/eid2608.201097

10. Zhang H, Rostami MR, Leopold PL, Mezey JG, O'Beirne SL, Strulovici-Barel Y, Crystal RG (2020) Expression of the SARS-CoV-2 ACE2 receptor in the human airway epithelium. Am J Respir Crit Care Med. https://doi.org/10.1164/rccm. 202003-0541OC

11. Loeffelholz MJ, Tang YW (2020) Laboratory diagnosis of emerging human coronavirus infections-the state of the art. Emerg Microbes Infect 9(1):747-756. https://doi.org/10.1080/22221751. 2020.1745095

12. Winichakoon $P$, Chaiwarith R, Liwsrisakun C, Salee P, Goonna A, Limsukon A, Kaewpoowat Q (2020) Negative nasopharyngeal and oropharyngeal swabs do not rule out COVID-19. J Clin Microbiol. https://doi.org/10.1128/JCM.00297-20

13. Ora J, Puxeddu E, Cavalli F, Giorgino FM, Girolami A, Chiocchi M, Sergiacomi G, Federici M, Rogliani P (2020) Does bronchoscopy help the diagnosis in COVID-19 infection? Eur Respir J. https://doi.org/10.1183/13993003.01619-2020

14. Lentz RJ, Colt H (2020) Summarizing societal guidelines regarding bronchoscopy during the COVID-19 pandemic. Respirology 25(6):574-577. https://doi.org/10.1111/resp.13824

15. Wahidi MM, Lamb C, Murgu S, Musani A, Shojaee S, Sachdeva A, Maldonado F, Mahmood K, Kinsey M, Sethi S, Mahajan A, Majid A, Keyes C, Alraiyes AH, Sung A, Hsia D, Eapen G (2020) American association for bronchology and interventional pulmonology (AABIP) statement on the use of bronchoscopy and respiratory specimen collection in patients with suspected or confirmed COVID-19 infection. J Bronchol Interv Pulmonol. https:// doi.org/10.1097/LBR.0000000000000681

16. Pericàs JM, Hernandez-Meneses $\mathrm{M}$, Sheahan TP, Quintana E, Ambrosioni J, Sandoval E, Falces C, Marcos MA, Tuset M, Vilella A, Moreno A, Miro JM (2020) COVID-19: from epidemiology to treatment. Eur Heart J 41(22):2092-2108. https://doi. org/10.1093/eurheartj/ehaa462

17. Flaxman S, Mishra S, Gandy A et al (2020) Estimating the number of infections and the impact of non-pharmaceutical interventions on COVID-19 in 11 European countries: technical description update. Imperial College London, pp 1-35. https://doi.org/10. 25561/77731

18. Prokop M, Van Everdingen W, van Rees Vellinga T, Quarles van Ufford J, Stöger L, Beenen L, Geurts B, Gietema H, Krdzalic J, Schaefer-Prokop C, van Ginneken B, Brink M (2020) CORADS - a categorical CT assessment scheme for patients with suspected COVID-19: definition and evaluation. Radiology. https://doi.org/10.1148/radiol.2020201473

19. Garbino J, Soccal PM, Aubert JD, Rochat T, Meylan P, Thomas Y, Tapparel C, Bridevaux PO, Kaiser L (2009) Respiratory viruses in bronchoalveolar lavage: a hospital-based cohort study in adults. Thorax 64(5):399-404. https://doi.org/10.1136/thx.2008.105155

Publisher's Note Springer Nature remains neutral with regard to jurisdictional claims in published maps and institutional affiliations. 


\section{Authors and Affiliations}

\section{Caterina Barberi ${ }^{1,2}$. Elena Castelnuovo ${ }^{1,2} \cdot$ Andrea Dipasquale $^{1,3} \cdot$ Federica Mrakic Sposta $^{4} \cdot$ Giulia Vatteroni $^{1,4}$. Lorenzo Maria Canziani $^{1,3}$. Marco Alloisio ${ }^{1,5}$. Michele Ciccarelli ${ }^{1,6}$. Carlo Selmi ${ }^{1,7}$. Giorgio Maria Ferraroli ${ }^{1,5}$}

Caterina Barberi

caterina.barberi@humanitas.it

Elena Castelnuovo

elena.castelnuovo@humanitas.it

Andrea Dipasquale

andrea.dipasquale@humanitas.it

Federica Mrakic Sposta

federica.mrakic_sposta@humanitas.it

Giulia Vatteroni

giulia.vatteroni@humanitas.it

Lorenzo Maria Canziani

lorenzo.canziani@humanitas.it

Marco Alloisio

marco.alloisio@hunimed.eu

Michele Ciccarelli

michele.ciccarelli@humanitas.it

Giorgio Maria Ferraroli

giorgio_maria.ferraroli@cancercenter.humanitas.it
1 Department of Biomedical Sciences, Humanitas University, 20090 Pieve Emanuele, Milan, Italy

2 Department of Emergency Medicine, Humanitas Clinical and Research Center-IRCCS, 20089 Rozzano, Milan, Italy

3 Department of Internal Medicine, Humanitas Clinical and Research Center-IRCCS, 20089 Rozzano, Milan, Italy

4 Department of Radiology, Humanitas Clinical and Research Center-IRCCS, 20089 Rozzano, Milan, Italy

5 Department of Thoracic Surgery, Humanitas Clinical and Research Center-IRCCS, 20089 Rozzano, Milan, Italy

6 Department of Pulmonology, Humanitas Clinical and Research Center-IRCCS, 20089 Rozzano, Milan, Italy

7 Department of Rheumatology and Clinical Immunology, Humanitas Clinical and Research Center-IRCCS, 20089 Rozzano, Milan, Italy 